\title{
Evaluación del efecto regulador de Chrysoperla externa sobre mosca blanca Trialeurodes vaporariorum en tomate
}

\author{
Evaluation of the regulatory effect of Chrysoperla externa on \\ whitefly Trialeurodes vaporariorum in tomato
}

Mayerly Alejandra Castro L. ${ }^{1}$; John Wilson Martínez 0.; Mónica Yadira Dotor R. ${ }^{3}$

${ }^{1}$ Ingeniera Agrónoma, Grupo GMBC, Universidad Pedagógica y Tecnológica de Colombia, Tunja, Colombia, mayerlycastro@ hotmail.com.

2 Docente, MSc Ciencias Agrarias, Universidad Pedagógica y Tecnológica de Colombia, Tunja, Colombia, john.martinez@uptc. edu.co.

3 Docente, MSc. Fisiología Vegetal, Universidad Pedagógica y Tecnológica de Colombia, Tunja, Colombia, mydotorr@unal.edu.co.

Citar: CASTRO, M.; MARTINEZ, J.; DOTOR, M. 2016. Evaluación del efecto regulador de Chrysoperla externa sobre mosca blanca Trialeurodes vaporariorum en tomate. Rev. Cienc. Agr. 33(2): 43-54. doi: http://dx.doi.org/10.22267/ rcia.163302.51.

Recibido: Junio 30 de 2015.

Aceptado: Junio 09 de 2016.

\section{RESUMEN}

El tomate Solanum lycopersicum L es una hortaliza de gran importancia en el ámbito nacional. Dentro de las principales limitantes de este cultivo se encuentra la mosca blanca, Trialeurodes vaporariorum Westwood (Hemíptera: Aleyrodidae). Este estudio evaluó en laboratorio la capacidad reguladora de Chrysoperla externa Hagen (Neuroptera: Chrysopidae) sobre mosca blanca T. vaporariorum. A partir de plantas infestadas con T. vaporariorum, se tomaron foliolos con presencia de ninfas de la plaga y se depositaron en recipientes plásticos con tapa, adicionando un individuo en cada estado larval del depredador C. externa. Se emplearon densidades de presa de 50, 75 y 100 individuos en los dos instares iniciales del depredador y 100, 150 y 200 para el tercer instar, con un testigo con 50 individuos sin depredador. Se empleó un diseño completamente al azar en condiciones de laboratorio de $17,8^{\circ} \mathrm{C}$ y $67,5 \%$ de humedad relativa. Se observaron diferencias estadísticas significativas entre densidades de presa en los tres instares evaluados. Se encontró, para cada una de las densidades de presas de T. vaporariorum evaluadas en primer instar de C. externa, porcentajes de mortalidad de $96,8 \%, 80,5 \%$ y 56,4\%, respectivamente; para el segundo instar, mortalidades de $94,4 \%, 77,5 \%$ y 75,4\%, en su orden y para el tercer instar mortalidades de $99,1 \%, 90 \%$ y $76,6 \%$, respectivamente. De acuerdo con los resultados obtenidos, C. externa ejerce un efecto regulatorio, presentando una respuesta funcional tipo II en sus tres instares larvales, cuando actúa como depredador de ninfas de T. vaporariorum.

Palabras clave: predador, respuesta funcional, control biológico. 


\begin{abstract}
Tomato (Solanum lycopersicum L) is one of the most important vegetable crops in Colombia. Among the limiting factors to tomato crops, whitefly Trialeurodes vaporariorum Westwood (Hemíptera: Aleyrodidae) seems to be one of the most important. The objective of this study was to evaluate, under laboratory conditions, the regulatory capacity of Chrysoperla externa Hagen (Neuroptera: Chrysopidae) (predator) on T. vaporariorum. Leaflets nymphs (prey). Preys were obtained from tomato plants infested with T. vaporariorum and then placed into plastic containers with covers. Thereafter, one individual of the predator, in first, second or third instar, was introduced. We use prey densities of 50, 75 and 100 individuals for the first and second larval instar of the predator and 100, 150 and 200 individuals for the third larval instar. We added a control with 50 individuals without predator. A complete randomized experimental design was used with five replicates, with $17.8{ }^{\circ} \mathrm{C}$ and $67.5 \%$ of relative humidity. There were statistical differences between prey densities in all instars. In the first instar mortality percentages were $96.8 \%, 80.5 \%$ and $56.4 \%$, and in the second were $94.4 \%, 77.5 \%$ and $75.4 \%$, to 50,75 and 100 preys, respectively. On the other hand, the third larval instar showed $99.1 \%, 90 \%$ y $76.6 \%$ mortalities. According to the results, C. externa exhibited a regulatory effect, showing II type functional response for all three larval instars evaluated.
\end{abstract}

Key words: Predator, functional biological

control.

\section{INTRODUCCIÓN}

En Colombia el cultivo de tomate Solanum Lycopersicum L. es de gran importancia por su alto nivel de consumo, el departamento de Boyacá se caracteriza por ser uno de los principales productores a nivel nacional. Este cultivo presenta periodo vegetativo corto, uso intensivo de mano de obra e insumos, con altos costos de producción, grandes problemas fitosanitarios y amplias fluctuaciones de precios en el mercado (Vallejo, 1999).

Dentro de los principales limitantes fitosanitarias de este sistema de producción se encuentra la mosca blanca Trialeurodes vaporariorum Westwood, que causa daños directos e indirectos. Se alimenta por medio de la succión de savia, la cual trae consecuencias como perdida del vigor y manchas cloróticas en las hojas. La excreción de hidratos de carbono en las hojas, flores y frutos (Espinel et al., 2008) origina un crecimiento superficial de hongos, e igualmente, se reporta a T. vaporariorum como un vector eficaz de diversos virus (Jones, 2003; Suarez et al., 2015). Este insecto prefiere para alimentarse, plantas de familias taxonómicas como crucíferas, leguminosas, malváceas y solanáceas (García et al., 2007). La mosca blanca presenta durante su ciclo de vida diversos estados de desarrollo que incluyen huevo, cuatro instares ninfales y el adulto, con un periodo de tiempo total desde huevo hasta la emergencia del adulto de 23 a 28 días, dependiendo de los factores ambientales y biológicos presentes en los hábitats de desarrollo, presentan una metamorfosis denominada incompleta (Morales et al., 2006).

En estudios realizados para el control de mosca blanca se han empleado una buena cantidad de productos de síntesis química de los cuales algunos ingredientes activos no presentan efecto sobre este insecto (Cardona et al., 2001). Cuando se evaluó la resistencia de poblaciones de T. vaporariorum a insecticidas, se encontraron individuos con 
niveles de resistencia al insecticida metamidofos (organofosforado), niveles de tolerancia a cipermetrina (piretroide) y niveles de susceptibilidad a metomil (carbamato) (Cardona et al., 2001; García et al., 2007). De acuerdo con lo anterior, el control biológico de T. vaporariorum se presenta como una alternativa promisoria, con ventajas para los productores como la reducción en el uso de productos de síntesis química, lo cual a su vez, permite disminuir los costos de producción y la afectación al medio ambiente.

El manejo biológico se puede realizar mediante el empleo de organismos benéficos capaces de promover la regulación de poblaciones de insectos plaga; entre estos organismos se encuentran los Neurópteros, especialmente aquellos pertenecientes a la familia Chrysopidae. Esta familia es una de las más grandes dentro de dicho orden, con cerca de 2000 especies descritas (New, 1991; Salamanca et al., 2010), destacándose como importantes depredadores de insectos plaga. Chrysoperla spp. es un género considerado como excelente depredador generalista, con un gran potencial de control biológico (Salamanca et al., 2010). Chrysoperla externa Hagen es un depredador con un amplio rango de distribución geográfica (Gitirana et al., 2001), presentando un alto potencial de control biológico en insectos plagas ubicados en estas zonas (Dos santos et al., 2005). C. externa se destaca por su capacidad depredadora, su amplio rango de distribución, alta adaptabilidad a diversos ambientes, sobrevivencia a la cría en laboratorio y tolerancia a algunos insecticidas. En estudios realizados se reportan que este insecto se alimenta agresivamente de hasta 21 especies de insectos plaga de cultivos con importancia económica (Cadena et al., 2007).

De acuerdo con Albuquerque et al. (1994), las hembras de C. externa depositan gran cantidad de huevos sobre las hojas y ramas, de tal forma que se encuentren cerca de la presa; esta larva emerge después de tres a seis días y presenta tres estados de desarrollo, con una duración aproximadamente de dos a tres semanas; posteriormente, desarrolla un capullo para empupar, presentándose la emergencia de estos adultos entre diez a catorce días. Estos insectos son voladores por lo cual se observa su presencia en el atardecer y la noche (Monje et al., 2012).

Loera et al. (2001) señala que dentro del ciclo biológico de este insecto se destaca como depredador, el estado larval. El segundo estadio larval consume más alimento que el primero, pero sobresale el tercer estadio por su alta capacidad depredadora y mayor tasa de consumo; las larvas de este estadio son las más agresivas y consumen insectos de cuerpo blando como áfidos y trips, entre otros (Salamanca et al., 2010). Mientras tanto, la dieta alimenticia del estado adulto consiste principalmente en néctar, polen o miel producida por otros insectos (Loera et al., 2001, Salamanca et al., 2010).

Los modelos poblacionales asociados al control biológico, resultan bastantes complejos, sin embargo, se ajustan a la realidad, de manera que son capaces de predecir y describir los cambios en la densidad de los individuos en una población en un tiempo y espacio determinados (Vargas y Rodríguez, 2008). La respuesta funcional es un modelo que describe la relación entre el número de presas consumidas por un depredador en función de la densidad de la presa, en un espacio e intervalo de tiempo fijos. La respuesta funcional es así de gran importancia para cualquier descripción sobre depredación o parasitismo, debido a que el número de presas consumidas, determina el desarrollo, supervivencia y reproducción de estos controladores (García et al., 2007). Holling (1959) describió tres tipos de respuesta funcional que dependen de dos parámetros establecidos que corresponden a los denominados tiempo de manipulación (denotado como $T h$ ) y coeficiente de ataque (denotado como a) (Torrealba y Arcaya, 2014).

El objetivo de este trabajo fue evaluar la 
capacidad reguladora de C. externa sobre el estado larval de mosca blanca, T. vaporariorum, en condiciones de laboratorio, con el fin de determinar la interacción depredador-presa, que permita proyectar el uso de este controlador en el manejo integrado de esta plaga.

\section{MATERIALES Y MÉTODOS}

El estudio se llevó a cabo en las instalaciones de la Universidad Pedagógica y Tecnológica de Colombia (Tunja, Boyacá). En el laboratorio de control biológico, área de entomología, con condiciones de $17,8^{\circ} \mathrm{C}$ y humedad relativa de $67,5 \%$, se observó la capacidad depredadora de C. externa y en un invernadero de vidrio se realizó la multiplicación de T. vaporariorum en tomate, como fuente de individuos para las pruebas en laboratorio.

Obtención del material biológico. Se sembraron plantas de tomate Solanum lycopersicum L., previamente adquiridas de un proveedor del municipio de Sáchica, departamento de Boyacá, las cuales se aislaron con una tela tul y se infestaron con material vegetal que tenía presencia de adultos de mosca blanca T. vaporariorum; así se mantuvo una población permanente en las plantas infestadas y se evitó la diseminación de la mosca blanca a otras zonas cercanas donde pudiera causar daño. Cuando se presentó una incidencia del $80 \%$ de individuos en estado ninfa de mosca blanca en las plantas de tomate, se seleccionó este material como fuente de individuos para las pruebas de depredación con $C$. externa en laboratorio.

Pruebas de depredación. La prueba de depredación se llevó a cabo en condiciones de laboratorio. Se tomaron foliolos de tomate con presencia de ninfas de mosca blanca, obtenidos en invernadero, y se llevaron al laboratorio. Allí, para evitar la deshidratación de los foliolos, se introdujo la base de cada uno de estos, en un frasco de vidrio de $5 \mathrm{ml}$ de capacidad, el cual contenía Agar-Agar en concentración de $13 \mathrm{~g} . \mathrm{l}^{-1}$ (para mantener el potencial de soluto debajo del 50\%); posteriormente los frascos se sellaron, con papel Parafilm y se depositaron en recipientes de plástico rectangulares de 32 onzas de capacidad.

El diseño estadístico utilizado fue completamente al azar, con cuatro tratamientos de los cuales se replicaron diez veces cada uno, cada replica representaba la unidad experimental, que consistió en un foliolo de tomate con presencia de ninfas de $T$. vaporariorum y se adicionó un individuo en estado larval del depredador C. externa. Los tratamientos buscaron evaluar la respuesta depredador-presa (respuestas funcional) de cada uno de los instares larvales de C. externa sobre ninfas de T. vaporariorum, por lo cual se ofrecieron densidades de 50, 75 y 100 presas para el primer y segundo instar larval del predador. En el tercer instar larval, por su mayor capacidad de consumo observada en pruebas preliminares, se proporcionaron densidades de 100, 150 y 200 individuos de la presa. Se incluyó un testigo absoluto con 50 individuos de T. vaporariorum, sin el depredador, para establecer una posible mortalidad natural en la población por la manipulación o las condiciones ambientales.

Pasadas 24 horas de la infestación, se iniciaron las observaciones, mediante el conteo de individuos de la presa T. vaporariorum ubicados en el envés de los foliolos de tomate y consumidos por el depredador. Las variables evaluadas fueron: número diario de presas consumidas y porcentaje de mortalidad causado por el depredador sobre la población de la presa a través del tiempo de observación.

Los resultados se analizaron con el programa SAS (SAS, 2001). Se realizó Análisis de Varianza (ANDEVA) para detectar diferencias entre tratamientos, verificando previamente los supuestos de homogeneidad de varianzas y distribución normal de residuos. Para la comparación de medias se utilizó la prueba de Tukey, con un nivel se significancia de $\alpha=0,05$. Se aplicaron los modelos matemáticos de Holling (1959) (ecuación del disco) a los datos de respuesta de depredación, usando un análisis de regresión por medio del paquete estadístico Statgraphics plus 5.1 . 


\section{RESULTADOS Y DISCUSIÓN}

Consumo diario. En relación con el consumo diario promedio de C. externa instar I, II y III, suministrándole diferentes cantidades de presa, se encontraron diferencias significativas $(\mathrm{P}<0,05)$ entre todos los tratamientos (Tabla 1).

La relación depredador presa en el instar larval I de C. externa, de acuerdo con el número de individuos ofrecidos, registró diferencias significativas; siendo mayor el consumo diario en el tratamiento de 75 presas ofrecidas $(15,1 \pm 0,22)$. Esto es similar a lo que demuestra Auad et al. (2005), con un consumo diario de 13,8 $\pm 1,17$ individuos de $B$. tabaci biótipo $B$, pero difiere de lo expresado por Dos santos et al. (2003) en cual obtuvieron un consumo de 6,3 $\pm 0,55$ con Aphis gossypii Glover y que contrasta con lo hallado por Dos santos et al. (2005) con un valor aproximado de 8,0 $\pm 1,50$ para la misma presa. Estos valores varían de acuerdo a la especie ofrecida debido a que tienen diferente tamaño, movilidad y aporte nutricional, lo cual influye en el desarrollo completo del depredador (Tabla 1).

En el instar II de C. externa, se presenta una mayor capacidad de depredación respecto al primer estadio, este resultado presenta una clara visión de lo expresado por Dos santos et al. (2003) quienes muestran en este instar un consumo diario de 20,0 \pm 2,12 individuos de Aphis gossypii Glover.
Este comportamiento esta dado en virtud que este instar requiere de un mayor rango de presas, para cubrir sus exigencias nutricionales y lograr así un desarrollo completo. Estos resultados son inferiores a los registrados por Auad et al. (2005), con un consumo diario de 28,8 $\pm 2,28$ individuos de $B$. tabaci biótipo B y Dos santos et al. (2005) con un valor de 28,1 $\pm 1,52$ para Aphis gossypii Glover. El menor nivel de consumo observado en este estudio está relacionado con la temperatura a la cual se trabajó el ensayo $\left(17^{\circ} \mathrm{C}\right)$, ya que los otros estudios mencionados anteriormente fueron conducidos a $25^{\circ} \mathrm{C}$, lo cual afecta directamente el metabolismo del insecto, requiriendo así el consumo de un menor número de presas por parte del depredador Oliveira et al. (2010) (Tabla 1).

El consumo diario de $C$. externa en el instar III en todos los tratamientos fue mayor con respecto a los instares anteriores. Esto se relaciona directamente con el mayor tamaño y mayor necesidad de alimento de las larvas de instares avanzados (Bastidas et al. 2010). Lo anterior permite verificar, la influencia entre la densidad de presa y la capacidad de depredación de $C$. externa. Por ser el III instar el de mayor actividad de consumo como depredador, sería el más apropiado para su implementación como una estrategia de control biológico, teniendo en cuenta sincronizar los ciclos de vida del depredador y de la presa, para garantizar este alto nivel de consumo (Tabla 1).

Tabla 1. Consumo diario de C. externa - instar I, II y II, con diferentes cantidades de presas de T. vaporariorum.

\begin{tabular}{cccc}
\hline \multirow{2}{*}{$\begin{array}{c}\text { Cantidad de } \\
\text { presas }\end{array}$} & Instar I & Instar II & Instar III \\
\cline { 2 - 4 } & $12,1 \pm 0,19(\mathrm{C})$ & $11,8 \pm 0,30(\mathrm{C})$ & $\mathrm{Ne}$ \\
50 & $15,1 \pm 0,22(\mathrm{~A})$ & $15,2 \pm 0,33(\mathrm{~B})$ & $\mathrm{Ne}$ \\
75 & $14,1 \pm 0,33(\mathrm{~B})$ & $18 \pm 0,58(\mathrm{~A})$ & $16,5 \pm 0,1(\mathrm{C})$ \\
100 & $\mathrm{Ne}$ & $\mathrm{Ne}$ & $22,5 \pm 0,14(\mathrm{~B})$ \\
150 & $\mathrm{Ne}$ & $\mathrm{Ne}$ & $25,5 \pm 0,12(\mathrm{~A})$ \\
200 &
\end{tabular}

* Letras diferentes indican diferencias estadísticas significativas. Ne, no evaluado. 
Porcentaje de depredación en el tiempo. En la evaluación del efecto de larvas de C. externa instares I, II y III, sobre ninfas de mosca blanca T. vaporariorum, en condiciones controladas, se presentaron diferencias significativas entre todas las densidades evaluadas $(\mathrm{P}<0,05)$. Cuando se aplicó la prueba de Tukey, se estableció que hay tres grupos estadísticamente diferentes, en cuanto el porcentaje de mortalidad ocasionado 24 horas después de introducir el depredador. El testigo absoluto no presentó mortalidad natural o por manipulación.

Se verificó un incremento lineal en el número de presas de T. vaporariorum consumidas por $C$. externa, relacionado con el aumento en su densidad, hasta las 96 horas; estos resultados obtenidos en el instar I, se asemejan al estudio de Auad et al., (2005) quienes observaron este mismo comportamiento lineal, pero con ninfas de Bemisia tabaci biótipo B transcurridas las 96 horas de estudio. La relación depredador presa varía de acuerdo con la densidad de individuos ofrecidos, con un crecimiento lineal en el mismo periodo de tiempo (Figura 1).

En la Figura 2 se observa un comportamiento ascendente, casi lineal, en todos los tratamientos hasta las 72 horas (tres días) de observación, seguido de una desaceleración de la mortalidad de T. vaporariorum por consumo del II instar de C. externa, respecto a los días anteriores, lo cual indica un consumo menor cuando está por finalizar el instar II después de 72 horas; este resultado contrasta con el obtenido por Auad et al. (2005), donde las larvas después de las 48 horas, tuvieron un consumo menor de presas en todos los tratamientos, empleando ninfas de B. tabaci biótipo B.

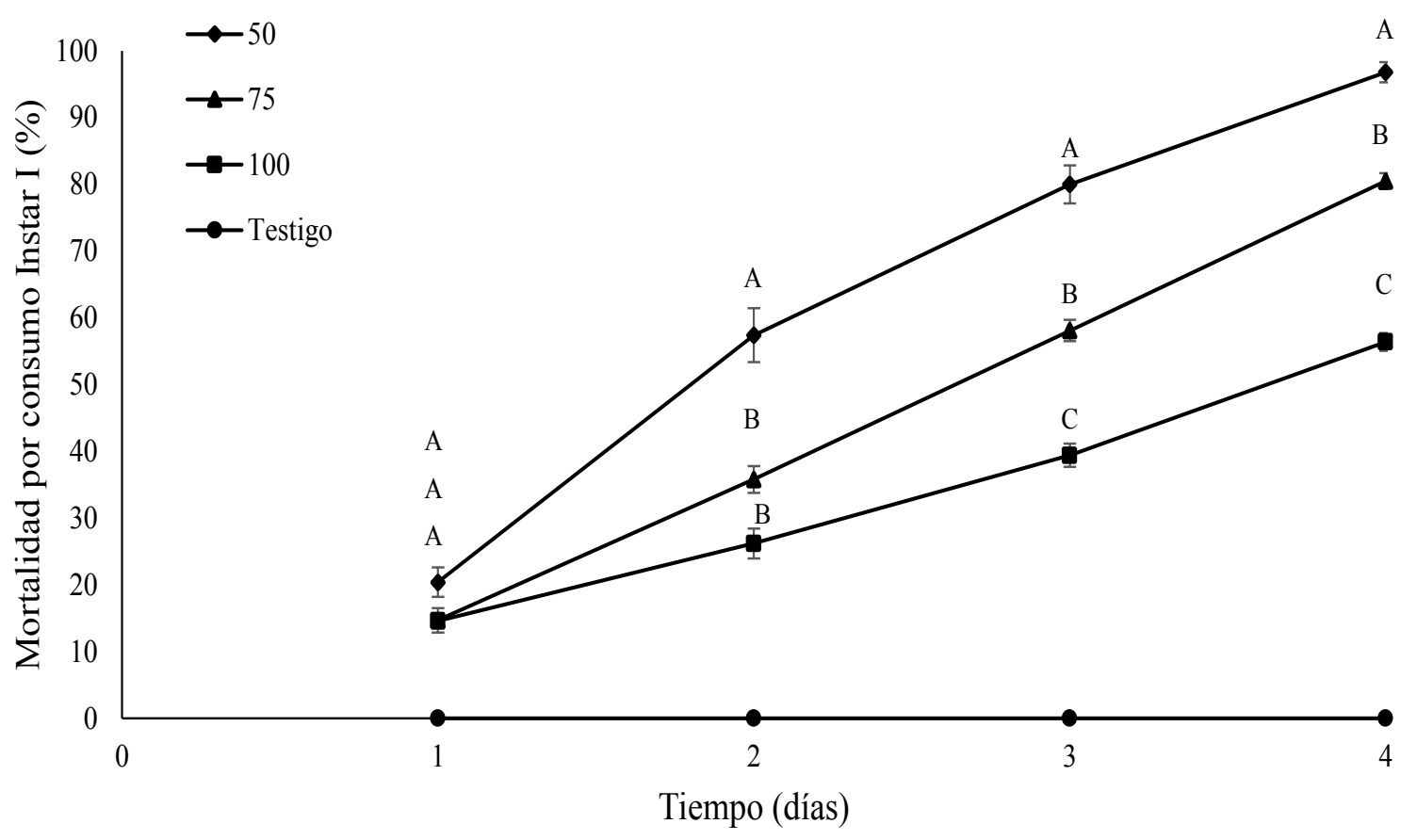

Figura 1. Porcentaje de mortalidad en T. vaporariorum causada por depredación del primer instar larval de C. externa a diferentes cantidades de presas. 


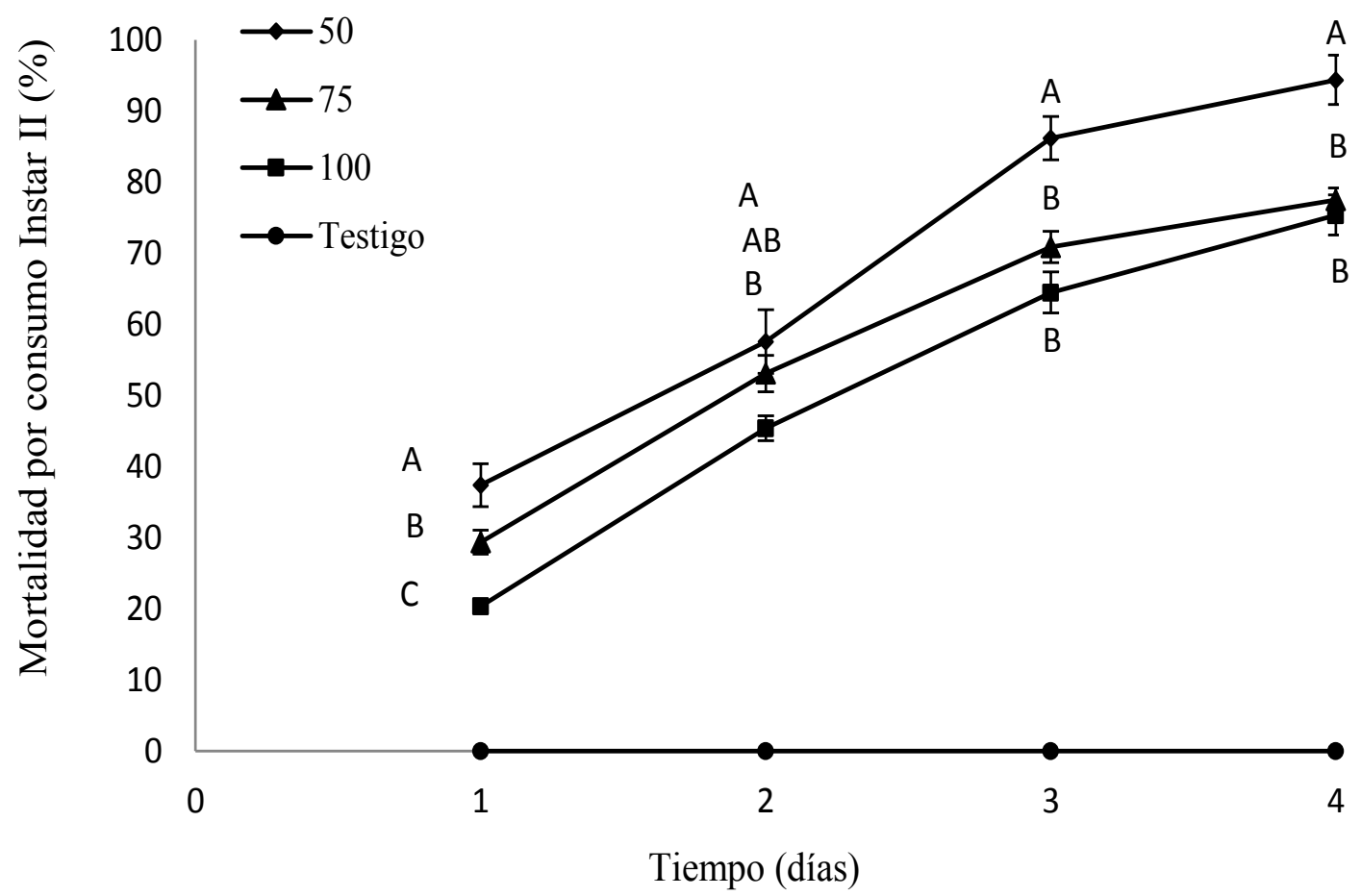

Figura 2. Porcentaje de mortalidad en T. vaporariorum causada por depredación del segundo instar larval de C. externa, a diferentes cantidades de presas.

Según como se observa en la Figura 3, se presenta un aumento lineal en el número de presas T. vaporariorum consumidas con respecto al tiempo transcurrido del ensayo, presentando una capacidad de depredación mayor que el primer instar larval, el anterior comportamiento se debe a que el segundo instar larval $C$. externa presenta mayor tamaño, mayor movilidad, lo que favorece una área de búsqueda mayor. Un comportamiento similar con ninfas de B. tabaci biótipo B fue descrito por Auad et al. (2007).

Por otro lado, el incremento de depredación de $C$. externa en el instar III, lo corroboran diferentes estudios, en los cuales se ofrecieron presas como Aphis gossypii, (Schlick-Souza et al., 2011) e individuos de Neohydatothrips signifer (Bastidas et al., 2010). Los autores antes citados evidenciaron un consumo mayor en este instar larval de $C$. externa, en comparación con los anteriores, como consecuencia del aumento de la cantidad de presas ofrecidas, lo cual favorece un mayor número de encuentros entre las larvas del depredador y sus presas, por lo tanto, se presentó una mayor depredación (Barbosa et al., 2008) (Figura 3).

Respuesta de depredación de C. externa sobre T. vaporariorum. Las variables que permiten expresar la respuesta funcional de un depredador incluyen la tasa instantánea de ataque (a) o eficiencia de búsqueda, que expresa la actividad y la capacidad que presenta el depredador de encontrar su presa, y el tiempo de manipulación (Th) que corresponde al periodo que el depredador gasta en atrapar, matar, consumir y digerir la presa.

La tasa instantánea de ataque del instar III de $C$. externa, fue mayor, en comparación con los otros instares evaluados, presentando una relación inversa con el tiempo de manipulación, como se observa en la Tabla 2. 


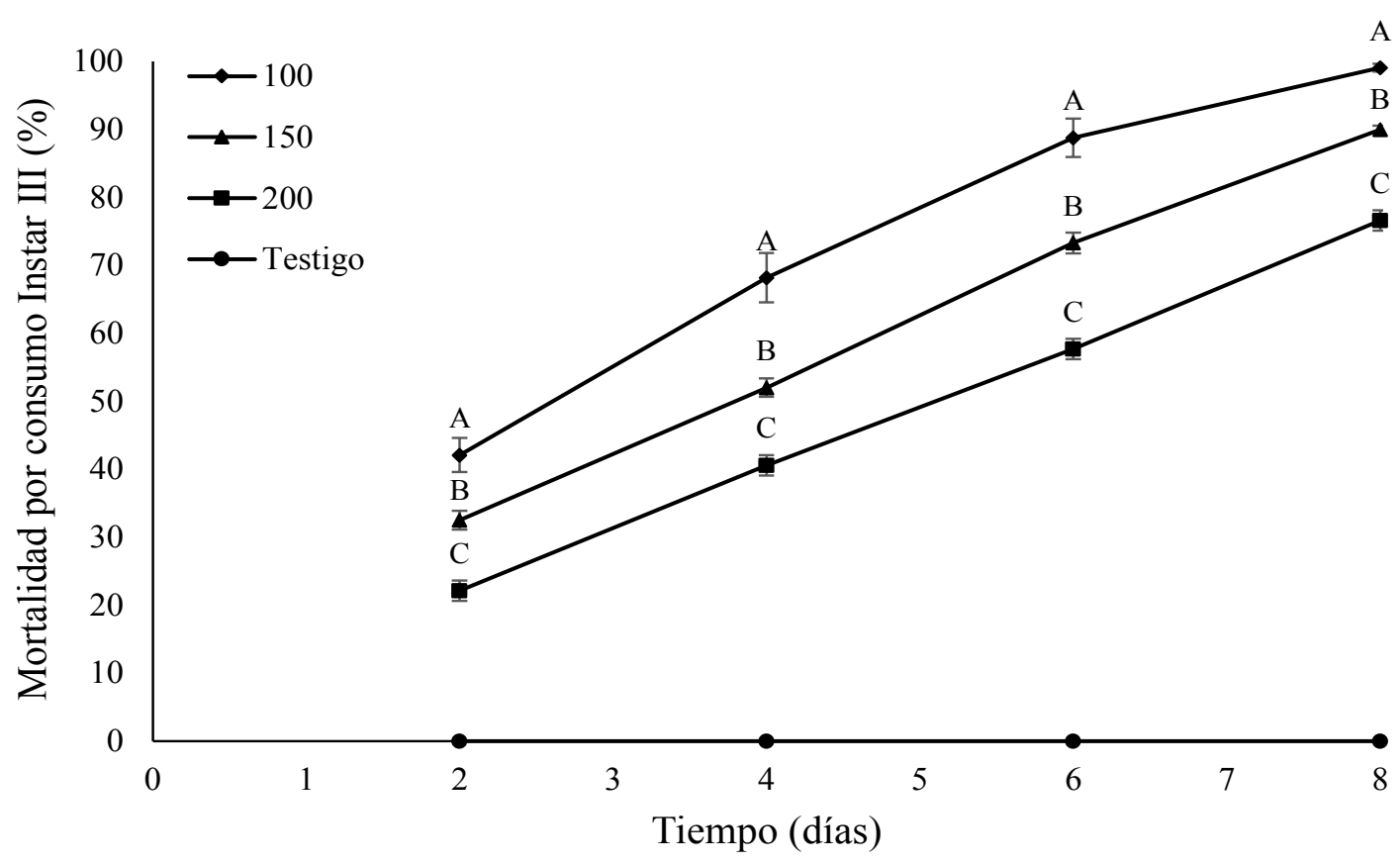

Figura 3. Porcentaje de mortalidad de T. vaporariorum causado por depredación del tercer instar larval de C. externa, a diferentes cantidades de presas.

Tabla 2. Respuesta funcional tipo II estimados con el modelo de Holling, para los tres instares larvales de C. externa sobre ninfas de T. vaporariorum.

\begin{tabular}{lcc}
\hline \multirow{2}{*}{ INSTAR LARVAL } & \multicolumn{2}{c}{ VARIABLES } \\
& $\mathbf{a}$ & Th \\
\hline Instar I & 0,036 & 0,240 \\
Instar II & 0,069 & 0,130 \\
Instar III & 0,097 & 0,062 \\
\hline
\end{tabular}

El valor 'a' del tercer instar resultó mayor que los obtenidos para el primer y segundo instar, mientras que el 'Th' fue menor en comparación con los dos anteriores instares, indicando que este instar presenta una mayor capacidad para encontrar su presa y puede manipularla más fácilmente, por tal razón, puede consumir un número mayor de ninfas.

Lo anterior se puede deber al tamaño de las crisopas de III instar, debido a que a mayor tamaño, mayor coeficiente de búsqueda, mayor el requerimiento de alimento y por ende una reducción en el tiempo de manipulación. Este periodo es un factor importante porque influye directamente en la cantidad de presas consumidas como lo afirma AilCatzim et al. (2012) quienes en su estudio con el tercer instar de $C$. carnea, presentó el mismo comportamiento con ninfas de $B$. cockerelli, al igual que Torrealba y Arcaya (2014) con P. clavatus, controlando al áfido negro del matarratón A. craccivora.

La evaluación de la capacidad depredadora de los tres instares larvales de C. externa, reveló un aumento en el consumo de presas a medida que avanzaba el desarrollo larval, en cada una de las densidades evaluadas. En el primer y segundo instar larval del depredador hubo una tendencia de estabilización en el consumo de T. vaporariorum con un valor máximo de 55 presas consumidas para el instar I y de 60 presas para el instar II, como se observa en la Figura 4. En el tercer instar, la larva presenta una mayor capacidad de depredación, con un consumo máximo de 129 individuos de los 200 ofrecidos, presentando una tendencia de estabilización desde las 100 presas, como se observa en la Figura 5. 


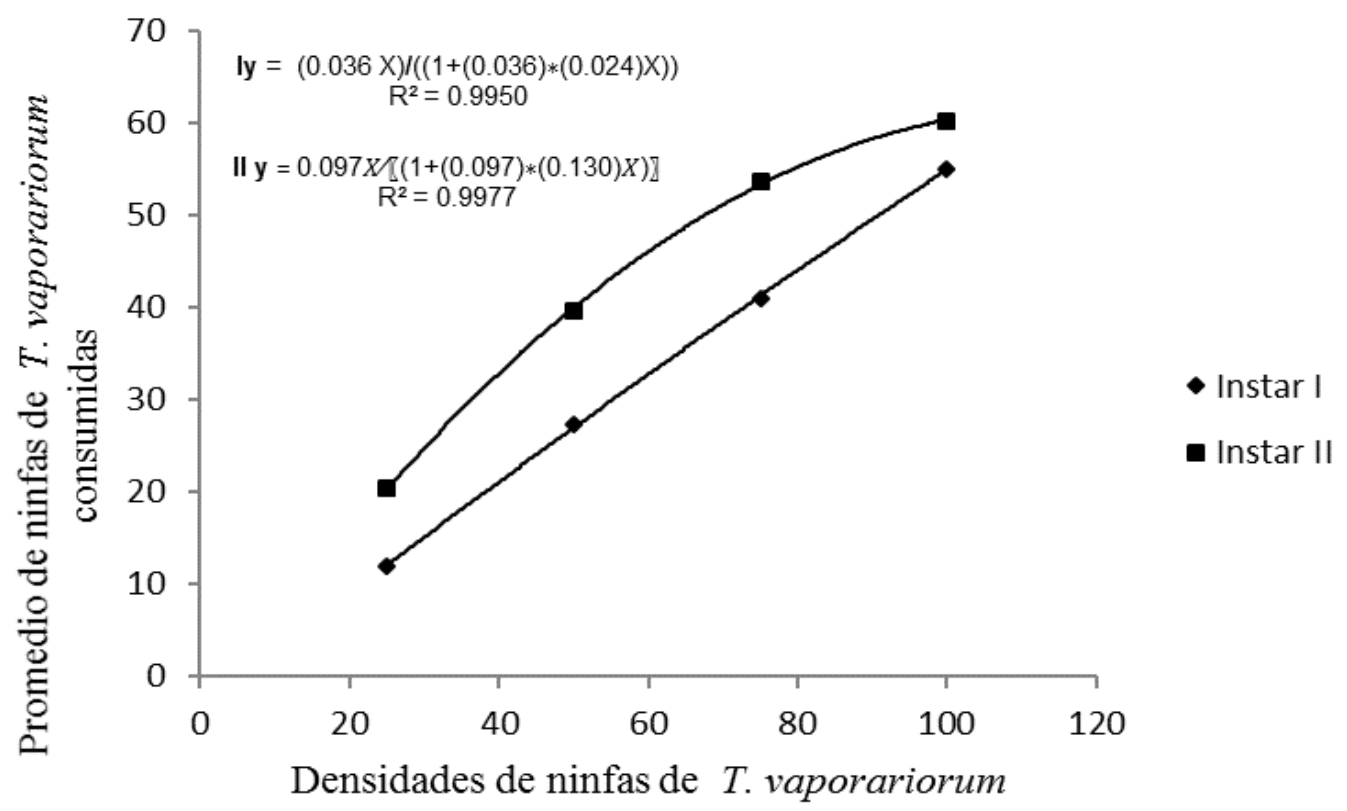

Figura 4. Respuesta funcional del primer y segundo instar larval de $C$. externa con varias densidades de ninfas de T. vaporariorum.

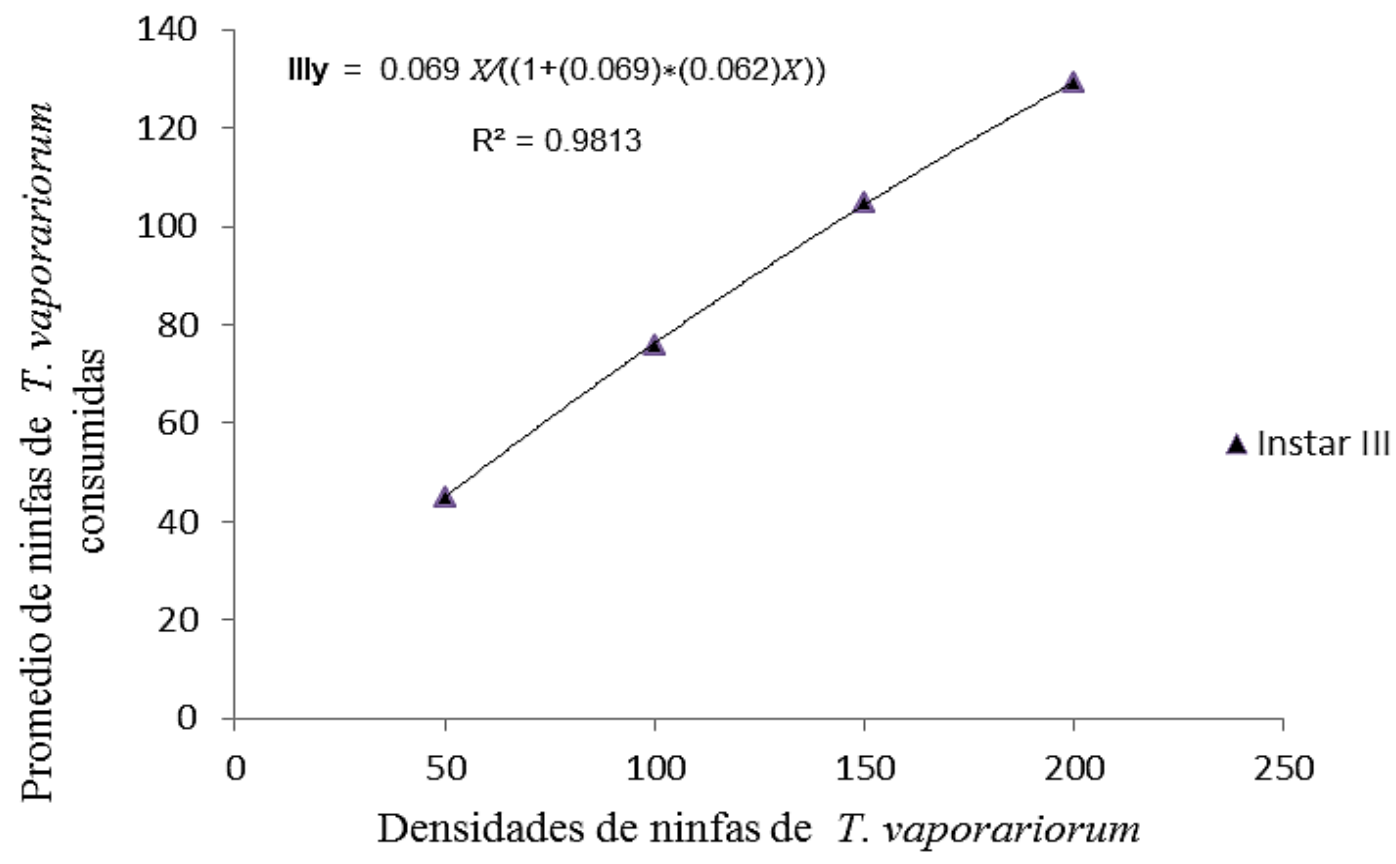

Figura 5. Respuesta funcional del tercer instar larval de C. externa con varias densidades de ninfas de T. vaporariorum. 
Chrysoperla externa Hagen se comporta como un depredador que se ajusta al tipo de curva II, donde la respuesta resulta en un aumento desacelerado a medida que se incrementan las presas consumidas, hasta llegar a una asíntota en la cual se expresa la máxima tasa de ataque (Hassell, 2000). Este comportamiento se observa más en el Instar II, dado que en el primer instar su tasa de consumo es menor comparado con el segundo, estos resultados concuerdan con Auad et al. (2005) para C. externa y Ail-Catzim et al. (2012) con C. carnea sobre ninfas de B. cockerelli, con una respuesta funcional tipo II para los tres instares del depredador.

Para el primer instar de $C$. externa se descarta que presente otro tipo de respuesta funcional debido a que para clasificarla en tipo I la densidad de la presa debe ser directamente proporcional a las consumidas por el depredador hasta llegar a un punto de estabilización y para el tipo III el depredador ataca cuando la población de la presa aumenta generando una mortalidad denso independiente directa (Fernández y Corley, 2004) (Figura 4).

El tercer instar larval presenta un alto grado de depredación, respecto a los instares anteriores, con una tasa de consumo mayor. Resultados semejantes fueron obtenidos por Torrealba y Arcaya (2014), Salamanca et al. (2010) y Dos Santos et al. (2005), con un mayor potencial de depredación en el instar III, que se puede explicar debido a que se presenta una mayor exigencia nutricional y mayor movilidad, propiciando una mayor área de búsqueda de la presa en este instar (Auad et al., 2005) (Figura 5).

En el análisis de la respuesta funcional del depredador aplicando el modelo de Holling se obtuvo un valor de F altamente significativo, con coeficientes de determinación de $\mathrm{R}^{2}$ para el primer, segundo y tercer instar larval con valores de 0,9950, 0,9977 y 0,9813 respectivamente; lo anterior indica que existe correlación entre el número de presas T. vaporariorum ofrecidas como presa y el número de estas consumidas por C. externa.

\section{CONCLUSIONES}

El porcentaje de depredación en el tiempo con diferentes cantidades de presa de Trialeurodes vaporariorum (Westwood) fue menor con las mayores densidades de presa (100 y 200), lo cual indica que este rango de presas es suficiente para suplir los requerimientos nutricionales del depredador Chrysoperla externa (Hagen).

La capacidad depredadora de las larvas de C. exter$n a$ en sus tres instares larvales, sobre ninfas de $T$. vaporariorum, fue menor en este estudio respecto a otras investigaciones, lo cual probablemente se debió a que la temperatura a la cual se trabajó fue menor que en los trabajos reportados.

Los estadios larvales de Chrysoperla externa (Hagen) presentaron una respuesta funcional tipo II, lo cual indica que el consumo aumento con el incremento en la densidad presas de Trialeurodes vaporariorum (Westwood), pero luego se presentó un comportamiento desacelerado a medida que se incrementó las presas consumidas, hasta llegar a un consumo constante.

De acuerdo con las observaciones realizadas de la capacidad depredadora de Chrysoperla externa (Hagen), en condiciones de laboratorio, se puede inferir que este insecto constituye una herramienta potencial de control biológico para su implementación en el manejo de Trialeurodes vaporariorum (Westwood) en el cultivo de tomate.

\section{REFERENCIAS BIBLIOGRÁFICAS}

AIL-CATZIM, C.; FLORES, M.; CERNA, E.; BADII, M.; LANDEROS, J.; OCHOA, Y.; AGUIRRE, L. 2012. Respuesta funcional de diferentes instares larvales de Chrysoperla carnea (Stephens) (Neuroptera: Chrysopidae) sobre ninfas de Bactericera cockerelli (Sulc) (Homoptera: Psyllidae). Rev. Fca Uncuyo. 44(2):279 - 288.

ALBUQUERQUE, G.; TAUBER, C.; TAUBER, M. 1994. Chrysoperla externa (Neuroptera: Chrysopidae): Life history and potential for biological control in Central and South. 4(1):8 - 13. 
AUAD, A.; CARVALHO, C.; SOUZA, B.; TREVIZANI, R.; FONSECA, C.; MAGALHÃES, R. 2005. Desenvolvimento das fases imaturas, aspectos reprodutivo e potencial de predação de Chrysoperla externa (Hagen) alimentada con ninfas de Bemisia tabaci (Gennadius) biótipo B em tomateiro. Acta Scientiarum. Biological Sciences. 27(2):327 - 334.

AUAD, M.; CARVALHO, C.; SOUZA, B.; SIMÕES, A.; OLIVEIRA, S.; FURTADO, A.; BOTELHO, R. 2007. Potencial de Chrysoperla externa (Hagen) no controle de Bemisia tabaci (Gennadius) biótipo B em tomateiro. Acta Scientiarum Agronomy. 29(1):29 - 32.

BARBOSA, L.; CARVALHO, C.; SOUZA, B.; AUAD, A. 2008. Eficiência de Crhysoperla externa (Hagen, 1861) (Neuróptera: Chrysopidae) no controle de Myzus persicae (Sulzer, 1776) (Hemiptera: Aphididae) em pimentáo (Capsicum annum L.). Ciênc. agrotec. Lavras. 32(4):1113 - 1119.

BASTIDAS, J.; DEVIA, D.; SANTOS, 0. 2010. Cría y evaluación de la capacidad de depredación de Chrysoperla externa sobre Neohydatothrips signifer, trips plaga del cultivo de maracuyá. Rev. Corpoica Cienc. Tecnol. Agropecu. 11(1):31 - 40.

CADENA, P.; ÁNGEL, F.; GÓMEZ, L.; GONZÁLEZ, R. 2007. Diferenciación morfológica y molecular de especies de Crisópidos (Neuróptera: Chrysopidae). Rev. Colomb. Entomol. 33(2):171 - 177.

CARDONA, C.; RENDÓN, F.; GARCÍA, J.; LÓPEZ-ÁVILA, A.; BUENO, J.; RAMÍREZ, J. 2001. Resistencia a insecticidas en Bemisia tabaci y Trialeurodes vaporariorum (Homoptera: Aleyrodidae) en Colombia y Ecuador. Rev. Colomb. Entomol. 27:33 - 38.

DOS SANTOS, T.; BOIÇA, A.; BARBOSA, J. 2005. Reposta funcional de Chrysoperla externa a Aphis gossypii em cultivares de algodoeiro. Manejo Integrado de Plagas y Agroecología. 74:41 - 47.

DOS SANTOS, T.; BOIÇA, A.; SOARES, J. 2003. Influência de tricomas do algodoeiro sobre os aspectos biológicos e capacidade predatória de Chrysoperla externa (Hagen) alimentada com Aphis gossypii glover. Bragantia. 62(2):243 - 254.
ESPINEL, C.; LOZANO, D.; VILLAMIZAR, L.; GRIJALBA, E.; COTES, M. 2008 Estrategia MIP para el control de Bemisia tabaci (Hemiptera: Aleyrodidae) en melón y tomate. Rev. Colomb. Entomol. 34(2):22 - 27.

FERNÁNDEZ, V.; CORLEY, J. 2004. La respuesta funcional: una revisión y guía experimental. Ecología Austral. 14:83 - 93.

GARCÍA, J.; BENÍTEZ, E.; LÓPEZ, A. 2007. Efecto de la densidad de población de Trialeurodes vaporariorum (Hemiptera: Aleyrodidae) sobre la eficiencia del depredador Delphastus pusillus (Coleoptera: Coccinellidae). Rev. Corpoica Cienc. Tecnol. Agropecu. 8(2):17 - 21.

GITIRANA, J.; CARVALHO, C.F.; SOUZA, B.; SANTA, L.V. 2001. Fluctuação populacional de espécies de Ceraeochrysa Adams. 1982 (Neuroptera: Chrysopidae) en citros na região de Lavras - MG. Cienc. Agrotec. 25(3):550 $-559$.

HASSELL, M. 2000. The spatial and temporal dynamics of host-parasitoid interactions. Oxford University Press. Oxford.

HOLLING, CS. 1959. Some characteristics of simple types of predation and parasitism. Can. Entomol. 91:385 - 398

JONES, DR. 2003. Plant Viruses Transmitted by Whiteflies. Eur J Plant Pathol. 109 (3):195 - 219.

LOERA, J.; VARGAS, J.; LÓPEZ, J.; REYES, M. 2001. Uso y manejo de Chrysoperla carnea. En: http://www.inifap. gob.mx; consulta: agosto, 2014.

MONJE, B. DELGADILLO, D. GÓMEZ, J. VARÓN, E. 2012. Manejo de Neohydatothrips signifer Priesner (Thysanoptera: Thripidae) en maracuyá (Passiflora edulis f. flavicarpa Degener) en el departamento del Huila (Colombia). Corpoica Ciencia y Tecnología Agropecuaria, 13(1):21 - 30.

MORALES, F. CARDONA, C. BUENO, J. RODRÍGUEZ, I. 2006. Manejo de integrado de enfermedades de plantas causadas por virus transmitidos por moscas blancas. CIAT, DFID y Tropical White Fly IPM Project. 43 p. 
NEW, T. 1991. Neuroptera In: Naumann, I.D. et al. (Eds). The Insects of Australia. Division of Entomology Commonwealth Scientific and Industrial Research Organization. Cornell University Press. New York. 525 -542 p.

OLIVEIRA, S.; AUAD, A.; SOUZA, B.; SILVA, D.; CARVALHO, C. 2010. Effect of temperature on the interaction between Chrysoperla externa (Neuroptera: Chrysopidae) and Sipha flava (Hemiptera: Aphididae). Eur. J. Entomol. 107:183 - 188.

SALAMANCA, J.; VARÓN, E.; SANTOS, 0. 2010. Cría y evaluación de la capacidad de depredación de Chrysoperla externa sobre Neohydatothrips signifer, trips plaga del cultivo de maracuyá. Rev. Corpoica Cienc. Tecnol. Agropecu. 11(1):31 - 40.

SAS Institute. 2001. SAS User Guide: Statistical Analysis System, version 8.2. SAS Institute Inc. Cary, NC, USA.

SCHLICK-SOUZA, E.; TOSCANO, L.; SOUZA-SCHLICK, G.; MARUYAMA, W.; ANDRADE, A. 2011. Desenvolvimiento larval de Chrysoperla externa alimentada com Aphis gossypii provenientes de três cultivares de algodoeiro. Rev. Agrarian Dourados. 4(13):182 - 188.

SUAREZ, L.; DÍAZ, M.; RODRÍGUEZ, D.; CANTOR, F. 2015. Medición indirecta de la tasa de consumo de adultos e inmaduros de Trialeurodes vaporariorum (Hemiptera: Aleyrodidae) sobre fríjol. Acta biol. Colomb. 20(3):99 - 109.

TORREALBA, J Y.; ARCAYA, E. 2014. Respuesta funcional de la larva de Pseudodoros clavatus (Fabricius, 1794) (Diptera: Syrphidae) al áfido negro del matarratón Aphis craccivora Koch, 1854 (Hemiptera: Aphididae). Entomotropica. 29(1):9 - 16.

VALLEJO, F. A. 1999. Mejoramiento genético y producción de tomate en Colombia. Universidad Nacional de Colombia sede Palmira. 216 p.

VARGAS, R.; RODRÍGUEZ, H. 2008. Dinámica de poblaciones pp 99 -105. En: Ripa R, Larral P (eds) Manejo de plagas en paltos y cítricos. Instituto de Investigaciones Agropecuarias. Colección Libros INIA 23. $200 \mathrm{p}$. 